\title{
Formación de posgrado en Extensión Agraria: experiencias en Holguín, Cuba
}

\author{
Niurlys Rodríguez-González, Nelvis-Alipio Almaguer-Pérez y José-Manuel García-Arias
}

\section{RESUMEN}

El extensionista, como facilitador de procesos agrarios en Cuba, desempeña diversos roles que implican conocimientos y habilidades. Sin embargo, la formación posgraduada en Extensión Agraria constituyó una limitante potencial hasta la década de 1990. Se realizó una investigación no experimental con profesionales vinculados a actividades extensionistas. El objetivo del artículo es mostrar los avances en la formación posgraduada en extensión agraria en Holguín, Cuba. Los resultados muestran una composición heterogénea de perfiles profesionales de los graduados, se constató alta aplicabilidad de los conocimientos adquiridos en el desempeño profesional y la apropiación del enfoque sistémico.

Palabras clave: posgrado, formación profesional, extensión agraria, Cuba.

Niurlys Rodríguez-González

niurlys@uho.edu.cu Cubana. Ingeniera Agrónoma, Universidad “Vladimir I. Lenin", Cuba; Máster en Gestión ambiental, Universidad de Holguín, Cuba. Profesora auxiliar del Centro de Estudios para Agroecosistemas Áridos, Universidad de Holguín, Cuba. Temas de investigación: extensión agraria y la agroecología.

Nelvis-Alipio Almaguer-Pérez

nelvis@uho.edu.cu

Cubano. Ingeniero Agrónomo, Universidad de Granma, Cuba; Especialista en Extensión Agraria, Universidad de Holguín, Cuba. Profesor auxiliar del Centro de Estudios para Agroecosistemas Áridos, Universidad de Holguín, Cuba. Temas de investigación: extensión agraria.

José-Manuel García-Arias

Cubano. Máster en Didáctica de la Química, Universidad Pedagógica de Holguín, Cuba; Profesoral superior de Química, Universidad de Oriente de Santiago de Cuba. Profesor auxiliar del Departamento de Ciencias Agropecuarias, Facultad de Ciencias Naturales y Agropecuarias, Universidad de Holguín, Cuba. Temas de investigación: extensión agraria y bioestimulantes naturales para las plantas. 


\title{
Formação de pós-graduação em Extensão Agraria: experiências em Holguín, Cuba
}

\section{RESUMO}

O graduado, como facilitador de processos agrários em Cuba, desempenha diversas funções que implicam conhecimentos e habilidades. Porém, a formação pós-graduada em Extensão Agraria constituiu uma limitante potencial até a década de 1990. Se realizou uma pesquisa no experimental com profissionais vinculados a atividades de extensão. O objetivo do artigo é mostrar os avances na formação pósgraduada em extensão agraria em Holguín, Cuba. Os resultados mostram uma composição heterogénea de perfis profissionais dos graduados, se constatou uma alta aplicabilidade dos conhecimentos adquiridos no desempenho profissional e a apropriação do enfoque sistémico.

Palavras chave: pós-graduação, formação profissional, extensão agraria, Cuba.

\section{Postgraduate training in Agrarian Extension: experiences in Holguin, Cuba}

\begin{abstract}
The extension agent, as a facilitator of agrarian processes in Cuba, plays several roles that involve knowledge and skills. However, the postgraduate training in Agrarian Extension was a potential constraint until the 1990s. The authors carried out a non-experimental research with professionals linked to the extension agent's activities. The objective of the article is to show the advances in the postgraduate training in agrarian extension in Holguín, Cuba. The results show that despite the despite the heterogeneity of the graduates' professional profiles, a high applicability of the acquired knowledge in their professional performance and the appropriation of the systemic approach could be verified.
\end{abstract}

Key words: postgraduate, professional training, agrarian extension, Cuba. 


\section{Introducción}

La extensión agraria ha pasado de un enfoque verticalista de transferencia de tecnologías a un enfoque horizontal y participativo porque se impone la necesidad de valorar cada vez más a los agricultores como sujetos activos de su desarrollo (Landini, 2016b). Se impone por ello la búsqueda de soluciones a partir de la identificación de problemáticas que afectan el desarrollo agrícola de las fincas, lo que implica la asesoría en diversas disciplinas a los productores por parte de los extensionistas. Sin embargo, es importante resaltar la necesidad de un cambio de actitud tecnicista a un enfoque sistémico de la persona que asume la labor extensionista (AlmaguerPérez, 2015).

Las capacidades básicas del extensionista se difunden sobre fundamentos personales de cualidades humanas esenciales e insustituibles: la ética, la autoestima, el autocontrol, la responsabilidad, la honestidad, la sociabilidad, el respeto mutuo, la tolerancia, amor al trabajo y la capacidad de convivir, todo lo que se reafirma con la formación de los extensionistas (Ribamar, 2009; Landini, 2014). La educación de adultos brinda la posibilidad de capacitarse permanentemente, en función de la velocidad de generación de tecnologías y del requerimiento del sector productivo, siendo una demanda en constante crecimiento por parte de los diferentes actores del sector.

La extensión agraria en Cuba inicia en la provincia Holguín a partir de la década de los ochenta y se acentúa posteriormente con la asesoría de proyectos de colaboración con Francia (Marzin et al., 2014). Fue creada la ocupación de agente de extensión agraria en la Estación Territorial de Investigaciones Agropecuarias de Holguín y se comienza con la formación a través de cursos con temáticas extensionistas. Sin embargo, aparecieron nuevos retos con la aparición del agente de extensión, tales como el de incorporar nuevas competencias por las implicancias interdisciplinarias de su labor. Se impuso la necesidad de profesionales integrales sustentados en un sólido sistema complementario de educación posgraduada (Horrutinier, 2007; Rodríguez et al., 2014).

En atención a la formación extensionista en el país se fueron incorporando otras modalidades de posgrados con la creación en 2006 de la maestría y la especialidad en Extensión Agraria en la Universidad Agraria de La Habana como centro rector. Posteriormente se inicia la especialidad en Extensión Agraria en su primera edición en Holguín, rectorada por la Universidad "Oscar Lucero Moya”. En este sentido, es importante abordar las demandas formativas, los principales resultados y las perspectivas. Por lo que el presente trabajo tiene como objetivo evidenciar los avances en la formación posgraduada en Extensión Agraria, a partir de la experiencia de los egresados en Holguín.

El artículo se organizó primero con algunas consideraciones teóricas de la extensión agraria, un segundo apartado sobre la metodología e instrumentos utilizados y por último se muestran los resultados y las conclusiones referentes al tema tratado.

\section{Algunas consideraciones teóricas sobre extensión agraria}

La definición del término extensión agraria ha resultado polémico porque se pueden encontrar definiciones que ponen énfasis en diferentes factores, como el social, el educacional o el transferencista. Engel (2001) define la extensión: "como un instrumento para fortalecer la capacidad de autoaprendizaje e innovación de las comunidades rurales hacia la competitividad y la sostenibilidad, prefieren una expresión más amplia que el apoyo técnico y la conceptualizan como proceso integral”.

\section{Origen y evolución en América Latina}

La extensión agraria y sus orígenes difieren entre países y continentes (McMahon et al., 2011). En América inicia en los Estados Unidos, en la década 1940-1950, y luego se disemina por varios países (Klerkx et al., 2016) y evoluciona en relación con los 
momentos histórico-sociales de cada país. En Argentina ayudó a generar las condiciones culturales y económicas para la "modernización", constituyó un arma poderosa para la transferencia de los paquetes tecnológicos de la "revolución verde" que consolidaron el uso industrial de los recursos naturales y justo aquí se perdieron los servicios como práctica pública (Enrique-Alemany, 2012). En Perú se ha producido una privatización parcial de los servicios de extensión, situación aprovechada por los actores privados para proporcionar asistencia técnica a través de casas comerciales y extensionistas particulares (Faure et al., 2015); igual situación ocurrió en Colombia, producto de la disminución del financiamiento para el servicio público de investigación, desarrollo y extensión (Henríquez, 2018).

Se ha mantenido como servicio público, como una de las misiones de institutos de investigaciones agropecuarias en varios países como Uruguay (Instituto Nacional de Investigación Agropecuaria), Paraguay (Instituto Paraguayo de Tecnología Agrícola), Bolivia (Instituto Nacional de Investigación Agropecuaria y Forestal), Ecuador (Instituto Nacional de Investigaciones Agropecuarias) y de igual manera para la mayoría de los países del Caribe y Centroamérica. En Haití y el Caribe anglófono las labores extensionistas son primordialmente financiadas por gobiernos donantes (Henríquez, 2018). Sobre el predominio de la visión transferencista Landini (2016a) corroboraba este hecho en encuesta aplicada a una muestra de extensionistas rurales de 10 países latinoamericanos (Argentina, Bolivia, Brasil, Chile, Ecuador, El Salvador, México, Paraguay, Perú y Uruguay), además resalta que se evidencia la carencia de articulación interinstitucional o con el apoyo a procesos de innovación rural.

\section{Concepto, origen y evolución en Cuba}

En Cuba, la extensión agraria es considerada como una disciplina emergente de los últimos 20 años. Su origen fue en 1950 con fines comerciales de maquinarias y agroquímicos, posteriormente se limitó a la transferencia de tecnologías. A partir de 1996, el Ministerio de la Agricultura (MINAG) inició un proceso de revisión y reorientación del Sistema Ciencia e Innovación Tecnológica Agraria (SINCITA), conformado con la mayoría de los centros de investigación agraria del país. En esos momentos se orientó al cambio de paradigma institucional de "ofertar resultados" por el de proyectar investigaciones por demandas del entorno debido a que se ha visto la corriente extensionista como sinónimo de capacitación, divulgación técnica o transferencia tecnológica (Caballero et al., 2000).

En 2001 fue consolidado el Sistema de Extensión Agraria (Marzin et al., 2014), pero en el país aún no ha sido institucionalizada la actividad extensionista (MINAG, 2011), a pesar de la necesidad latente. Es necesaria la valorización de fortalecer los sistemas públicos de extensión por el papel crucial que pueden desempeñar, para ello es necesario invertir (Wang et al., 2016).

Existen razones justificadas que demandan la consolidación de la extensión agraria en lo nacional, lo que se explica porque la producción de alimentos aún no satisface la demanda de la población (Riera et al., 2016), al mismo tiempo que el país importa gran cantidad de provisiones (Matías-González, 2012). También hay manifestaciones del cambio climático porque el frío ha disminuido durante el invierno y las sequías son más severas, faltan inversiones significativas y es baja la productividad. Además, se transita hacia una agricultura sostenible sobre bases agroecológicas (Vázquez et al., 2017).

\section{Formas y enfoques de extensión agraria}

En los últimos 15 años América Latina y el Caribe han experimentado muchos cambios. Entre los más importantes se encuentra una redefinición del rol del Estado, economías más abiertas y un aumento en el comercio internacional. El sector agrícola ha ido enfrentado estos cambios para seguir compitiendo en mercados globales y nacionales. 
Para que los sistemas de extensión contribuyan a la reducción de la pobreza es necesario pasar de la transferencia de tecnologías a otras formas (FAO, 2014). Uno de los grandes errores cometidos en el desarrollo agrario ha sido el enfoque reduccionista en las diferentes ramas y actores que tienen responsabilidades o tributan tecnologías o servicios a los escenarios productivos (Vázquez-Moreno, 2008). Se ha visto la transferencia de tecnologías como la extensión difusionista, única forma de extensión posible (Landini y Riet, 2015).

En Cuba adoptan diferentes posturas los profesionales vinculados a la extensión agraria debido a la coexistencia de diferentes formas extensionistas (Pavón, 2015). Una definición de las cuatro representaciones existentes fue dada por Marzin et al., (2003) como la forma transferencista, que consiste en transmitir conocimientos técnico-científicos a los productores; la desarrollistas elaborada por Paulo Freire, que considera la extensión como proceso educativo; la participativa, que moviliza los conocimientos técnicos científicos con una pedagogía adaptada, y la integral, aquella que rompe con el esquema lineal de transferencia de tecnología y comunicación por la respuesta real a las demandas de los agricultores.

\section{Breve revisión curricular sobre formación en extensión agraria}

La evaluación curricular es un proceso continuo que busca la mejora en función de sus estructuras en diferentes escenarios, teniendo en cuenta los cambios internos y externos (Culebro et al., 2017). La integración curricular unifica principios filosóficos y prácticos en el enfoque de enseñanza-aprendizaje indispensable tanto en la formación de profesionales o en el posgrado y se hace urgente ese binomio integrador de teoría-práctica en la formación en extensión agraria. En general, son muy limitadas las publicaciones científicas sobre extensión agraria y agricultura en Cuba (Rodríguez et al., 2016), lo que implica escasez documental en lo concerniente a evaluaciones curriculares a los programas formativos extensionistas.

En América Latina hay universidades donde la asignatura Extensión Agraria o Extensión Rural, aparece en los currículos de formación de los ingenieros agrónomos como obligatoria en países como Uruguay y Argentina, por citar ejemplos (Salguero et al., 2018). Sin embargo, los programas concebidos para la formación agronómica en Cuba vigentes desde 1900 no incluían la Extensión Agraria (Novo, 2011).

En 1999 las comisiones nacionales de carreras decidieron incorporar la Extensión Agraria al currículo de las carreras Ingeniería Agronómica y Mecanización Agropecuaria, porque hasta entonces predominó en la formación un enfoque técnico productivo y una limitada presencia de temáticas de corte humanístico en su currículo. Esto significaba graduar profesionales con elevado nivel técnico, pero poco preparados para asumir su función como promotor de un desarrollo rural sostenible, como demandan las nuevas condiciones del sector agrario cubano (Salguero et al., 2018).

Con la aparición del programa de la asignatura extensionismo agrícola en los currículo de pregrado se ha pretendido formar un profesional del sector capacitado para desenvolverse en actividades y situaciones diversas: reuniones de productores, funcionarios y técnicos; coordinación de grupos para resolver problemas técnicos y/o comunitarios (Sablón et al., 2012). El programa de la asignatura se construyó colectivamente en los talleres nacionales de profesores de Extensión Agraria de las universidades cubanas. Fueron incluidos tres temas (Extensión agraria y el desarrollo rural, Extensión como proceso de educación, Diagnóstico y adopción de tecnologías en el trabajo de extensión) y cada universidad asume la asignatura como obligatoria pero dentro del currículo propio y se imparte en $3^{\circ}$ o $4^{\circ}$ año de la carrera de Agronomía (Salguero et al., 2018).

Las carencias curriculares antes mencionadas hacen significativo que egresados antes de la inclusión 
de la asignatura tengan concepciones diferentes a los que se han graduado con posterioridad. Esta situación presupone la necesidad de la intensificación de la formación posgraduada con asignaturas propias de la extensión agraria (Miranda et al., 2016). Lo que exige que en el diseño curricular se tenga en cuenta la competencia profesional por ser correlacional, holística y contextual, basada en un enfoque de competencias como necesidad de responder adecuadamente al cambio social y tecnológico ocurrido y no a la adquisición de títulos (Vargas, 2008).

En el ámbito mundial, los estudios sobre la formación de posgrado se han orientado a la caracterización de problemáticas y al referenciamiento de preferencias que están haciendo repensar las acciones de universidades que se gobiernan por paradigmas del siglo XIX para las demandas del mercado del siglo XXI (Cruz, 2014). La importancia de los posgrados y la necesidad de la articulación con la investigación, el sector empresarial y la sociedad se considera como una tendencia emergente en la mayoría de los países de América Latina (Aguirre et al., 2019) y Cuba no está exenta de ello.

La formación en Extensión Agraria en América Latina ha tenido una fuerte influencia de la escuela norteamericana relacionada con las formas de extensión que han evolucionado según los procesos que han acontecido a nivel global. Sánchez-de-PuertaTrujillo (1996) denomina varios procesos a nivel internacional; expresa que antes de la revolución verde en Latinoamérica se disponía de expertos locales y posterior a ello se crean los servicios de extensión importados de los Estados Unidos, con lo cual también se importa la formación. Añade que la asistencia internacional en extensión a Latinoamérica se realiza por dos vías: asesoramiento de expertos asignados, y formación en el Centro Agrícola Internacional de Wageningen de Holanda y el Centro Internacional de Capacitación sobre Extensión Rural, dependiente del Servicio de Extensión Agraria de España. A partir de 1973, el personal de los servicios de Extensión
Agraria de Latinoamérica comenzó a acudir al centro internacional de España.

Almaguer-Pérez (1996) expresaba, sobre la experiencia de formación extensionista recibida en España y transferible a las condiciones de Cuba, que son necesarios reajustes en el contenido de los cursos. Este autor tomó como objeto de análisis el VII Curso Internacional de Extensión Agraria y Desarrollo Rural y consideraba que los cursos de Contabilidad pueden simplificarse, ya que son de mayor interés los referidos a métodos de investigación sociológica, comunicación, educación, recursos didácticos y teoría, enfoques, evolución e historia de la extensión.

El primer programa de educación de posgrado cubano surgió en 1992 y fue aprobado en el Consejo de Dirección del Ministerio de Educación Superior. Dicho programa contenía, como novedad, la inclusión de la figura de Maestría. De esta manera, en el nivel de posgrado se plantearon como formas organizativas: la autopreparación, el adiestramiento laboral, el curso, el entrenamiento, la especialidad de posgrado, la maestría y el doctorado. Actualmente el posgrado en Cuba está legislado por la Resolución 140/2019 en el artículo 31, y se definen como formas organizativas de la formación académica: la maestría, la especialidad de posgrado y el doctorado. Los programas se estructuran con un sistema de créditos (un crédito académico equivale a 30 horas) que se clasifican de acuerdo con los objetivos y la estrategia de formación. Estos programas incluyen cursos obligatorios, opcionales y libres. También queda establecido como necesario el análisis y dictamen de propuestas de modificaciones a los programas de especialidad de posgrado (Gaceta Oficial de Cuba, 2019). Porque la renovación del currículum de formación de las distintas carreras profesionales, ha constituido una exigencia asociada a las demandas de un mundo cambiante y al desarrollo de la sociedad del conocimiento (Castro et al., 2017).

En Cuba es urgente considerar modificaciones a los curricula de programas de posgrado en agricultura 
con la inclusión de herramientas extensionistas como alternativa potencial para formar competencias. La agricultura cubana impone de manera incuestionable estrechar los lazos entre la extensión agraria y la investigación agronómica; la primera carente de un sistema articulado, sistémico y generalista que refuerce la capacidad de los productores a mejorar su gestión como agricultor, tanto desde el punto de vista individual como colectiva; la segunda, en la mayoría de los casos, no conoce cuáles son las verdaderas demandas de los productores (Cid-Lazo, 2017).

Salguero et al. (2016) publicaron un artículo donde afirman que respecto a la formación posgraduada en Extensión Agraria los programas formativos están dirigidos a formar capacidades para investigaciones de desarrollo y articulación de actores, ya que en sus cursos e investigaciones se evidencia la creación de capacidades para actuar en función de situaciones problemáticas que se presentan en agroecosistemas, comunidades rurales o procesos de formación, desde el punto de vista social, ambiental y económico.

Como una disciplina emergente, la Extensión Agraria se posicionó en el posgrado a partir de 2006, con los programas de maestría y especialidad. Para la definición del programa de ambas modalidades de posgrado extensionista se desarrollaron talleres en los que tomaron como referentes las experiencias acumuladas. Los programas fueron aprobados por la Comisión Asesora para la Educación de Posgrado (COPEP) por medio de la resolución 314/2006 del Ministerio de Educación Superior (Salguero et al., 2016).

Es interesante sistematizar más las experiencias formativas y dar continuidad a evaluaciones de competencias en extensión agraria, porque evaluar competencias es recurrir a variedad de estrategias complementarias, como la carga académica y modalidades evaluativas que varían por curso y docente (Vargas, 2008). Actualmente, en Cuba se ha desarrollado la maestría sólo en la Universidad Agraria de la Habana, con varias ediciones, y la especialidad se ha impartido en la sede central e importado a cuatro Universidades (Pinar del Río, Camagüey, Holguín, Isla de la Juventud) en la nación cubana.

\section{La provincia de Holguín y la formación extensionista}

Con más de un millón de habitantes y un fuerte potencial agropecuario, la provincia de Holguín es pionera en la extensión agraria en la nación porque en ella surgió en 1987 la Estación Territorial de Investigaciones Agropecuarias de Holguín (ETIAH). Con la creación de esta nueva institución se aglutinaron varias estaciones de investigación y áreas experimentales (antes pertenecían a institutos nacionales de investigación) y aparecieron los planes de introducción de logros con mayor acercamiento a la base productiva.

Entre 1994 y 1997 se desarrolló un proyecto plurianual franco-cubano de apoyo a la renovación de los métodos de extensión agraria en Cuba. Posteriormente, entre 2001 y 2003, en las provincias de Holguín, Camagüey y La Habana se desarrolló el proyecto franco-cubano (PASEA) con el objetivo de coadyuvar al aumento de la producción agropecuaria a través de la introducción de métodos modernos para mejorar los mecanismos de la articulación ciencia-extensión- producción (Marzin et al., 2014). El PASEA contribuyó significativamente a la implementación del Sistema de Extensión Agraria (SEA) en el país y especialmente fue relevante para Holguín.

A pesar que mucho se ha avanzado desde la percepción manifestada por los profesionales vinculados a la actividad extensionista en la provincia, aún existe mucho por hacer. En la provincia, en el contexto territorial, prevalecen obstáculos que dificultan la gestión del conocimiento y la innovación (Hernández-Gil et al., 2014), entre los que se encuentran el verticalismo y sectoralismo en las relaciones institucionales; insuficiente preparación de los actores locales para realizar las acciones auto-transformativas que se requieren; presencia de métodos autoritarios en la conducción de acciones comunitarias, y el aún limitado espacio a 
proyectos de economía local y agentes económicos no estatales (Peña-Borrego et al., 2018).

Existen otros retos, como continuar cambiando el enfoque analítico prevaleciente aún por el sistémico. También el énfasis en la participación multiactores y la investigación-acción participativa porque como afirmaran Craps y Sips (2014), "investigación que sólo produce libros y artículos no es suficiente para cambiar una realidad". Aún más, se considera urgente el fortalecimiento de la formación en extensión agraria tanto en el pregrado como en el posgrado.

Las primeras acciones provinciales desde la Universidad de Holguín para la formación en Extensión Agraria comenzaron en el pregrado, con la carrera de Ingeniería Agrónoma en el curso 2009-2010 y en 2015 con la primera edición de Especialidad de posgrado. En este marco formativo, en la Facultad de Ciencias Naturales y Agropecuarias se creó la cátedra de Extensión Agraria Paulo Freire, con el objetivo de aglutinar a profesionales y estudiantes vinculados a la actividad agropecuaria, para construir un espacio de reflexión, actualización y formación e incidir sobre el desarrollo de los productores agropecuarios por medio de la investigación-acción.

\section{Metodología empleada}

La presente investigación toma en cuenta el periodo noviembre 2012 a diciembre 2018, en el cual se realizó la investigación descrita en este trabajo. La misma se desarrolló en cinco etapas, a saber:

Etapa 1. Síntesis y análisis de los inicios de la formación posgraduada. Se entrevistó a personas vinculadas a la labor extensionista desde su comienzo en Holguín, con el objetivo de recopilar las experiencias de enseñanza que fueron implementadas desde 1990 hasta 2012. Para sistematizar las experiencias de posgrado posteriores a 2012, el equipo coordinador de la primera edición de la especialidad en Extensión Agraria en Holguín compiló el proceso desde su gestación, desarrollo y terminación (periodo 2012-2018), lo que se describe en las etapas a continuación.

Etapa 2. Determinación de la demanda en la formación de posgrado (2012). Se realizaron encuentros informales con especialistas de diferentes formas de extensión agraria presentes en Holguín en entidades de investigación, enseñanza, producción a nivel provincial. En un segundo momento, se aplicaron entrevistas semiestructuradas para identificar temáticas de interés formativo en los profesionales vinculados a la actividad extensionista, pertenecientes a centros rectores de diferentes formas de extensión agraria: Unidad de Extensión, Investigación y Capacitación Agropecuaria de Holguín (UEICAH); Escuela de Capacitación de la Agricultura; también se contactó a seis especialistas y a agentes de extensión ubicados en cada uno de los 14 municipios de la provincia Holguín. La forma transferencista de tecnologías, con la Granja Urbana, empresas agropecuarias y delegación provincial de la agricultura, donde se intercambió con tres especialistas a nivel provincial, seis administradores de organopónicos y ocho especialistas de municipios que concurrieron al encuentro. La forma participativa, con la Asociación Nacional de Agricultores Pequeños (ANAP), donde se socializó la convocatoria con el presidente y dos instructores de zonas.

Etapa 3. Identificación y extrapolación de especialidad de posgrado en Extensión Agraria. A partir de búsqueda en la red nacional de universidades y contacto telefónico con profesionales vinculados a la Extensión Agraria de los ministerios de Educación Superior y de Agricultura, se identificó la Especialidad de Posgrado en Extensión Agraria en la Universidad Agraria de La Habana (UNAH). Una vez establecidos contactos formales y trámites con la Facultad de Agronomía, titular del programa, se importó a la Universidad de Holguín y fue oficializado a inicios de 2015.

Etapa 4. Desarrollo de los dos diplomados que conformaron la especialidad de posgrado Se adoptó 
la dinámica de desarrollo de los módulos sustentada en la construcción colectiva del conocimiento, con el profesor como facilitador, basados en los principios de la educación popular. Los cursos se adaptaron y rediseñaron sobre la base del contexto holguinero, a partir de ajustes demandados por los cursistas, por ejemplo, en la inclusión de entrenamiento práctico al concluir el diplomado dos.

Etapa 5. Sistematización de experiencias con egresados titulados de "Especialistas en Extensión Agraria". Se realizaron entrevistas semiestructuradas a los 29 graduados, para ejemplificar la incidencia de la formación recibida en sus puestos de desempeño profesional en los territorios. Se listaron las acciones emprendidas y agruparon en tres dimensiones:

- Herramientas de análisis: corresponden a diagnósticos en diferentes niveles de sistemas, o sea: municipio, sistema de cultivo o crianza. También la formulación participativa de planes de acción con agricultores en función de resolver problemáticas diversas.

- Procesos de participación y/o capacitación: implica herramientas de trabajo grupal, organización y facilitación de reuniones, así como procesos de capacitación multiactores.

- Inserción en investigaciones y/o innovaciones: se trata de acciones de investigación en fincas con agricultores, así como inventario y monitoreo de innovaciones agrícolas.

Procesamiento de la información. En las etapas uno y cuatro los datos fueron obtenidos mediante entrevistas semiestructuradas y en la tercera por consulta y listado de temas de investigación final. En todos los casos se elaboró una base de datos en Excel para Windows, se calcularon porcentajes, y se graficaron para visualizar las tendencias y hacer las correspondientes interpretaciones.

\section{Resultados \\ Inicio de la formación posgraduada (1990-2012)}

Fase 1: síntesis y análisis de los inicios. La formación extensionista en Cuba es algo reciente; a partir de la década de 1990 se inicia la formación de algunos profesionales con el apoyo de otros países. La primera experiencia fue con un investigador de la Estación Territorial de Investigadores Agropecuarios de Holguín (ETIAH) que recibió curso sobre capacitación agraria en Egipto. A partir de 1994 se inicia en la ETIAH un proyecto de investigación-formación-desarrollo cuyo objetivo esencial fue apoyar en aspectos metodológicos la implementación de un Sistema de Extensión Agraria en Cuba.

El primer proyecto franco-cubano dio inicio a varias modalidades de formación en el periodo 19941997 con la asesoría directa de un experto de la Universidad de las Antillas y Guyana de Guadalupe, bajo las metodologías de la escuela francesa de investigación-desarrollo. La formación se basó en cursos con clases teórico-prácticas, trabajo de terreno, labor de los profesionales formados hacia la enseñanza en el pregrado del Instituto Superior Pedagógico y de institutos politécnicos agropecuarios. Como salidas evaluativas se realizaron tesis de Agronomía con metodologías extensionistas desarrolladas por estudiantes universitarios y de los posgrados.

A partir de 1998 se intensifica la participación de extensionistas e investigadores en cursos de formación, entrenamientos e intercambios. Las acciones de posgrado implementadas se relacionan a continuación:

- Primer curso de posgrado en Extensión Agraria organizado por profesionales vinculados a esta actividad en la ETIAH (1998).

- Dos investigadores reciben formación en Extensión Agraria en la Escuela de Capacitación Agraria de España (1998). 
- Ejecución del segundo proyecto franco-cubano, Proyecto de Apoyo al Sistema de Extensión Agraria (PASEA), que garantizó con periodicidad mensual la formación continua de profesionales. Hubo implicancias desde la teoría y la práctica, se afianzaron conocimientos relacionados con la aplicación de los enfoques sistémicos, participativos y generalistas (2001 y 2003).

- Se organiza e imparte por profesionales de la ETIAH el diplomado denominado "Extensión Agraria para un desarrollo sostenible" (2004-2005).

- Continúa la formación extensionista con la incidencia de proyectos territoriales y nacionales. Se destacó el Programa de Innovación Agrícola
Local (PIAL) por la utilización del fitomejoramiento participativo y herramientas de análisis (2006-2012).

\section{Sistematización del proceso de la primera edición de la especialidad de posgrado en Holguín (2012-2018)}

Fase 2: determinación de la demanda en la formación de posgrado (2012). En encuentros directos en los espacios donde se investigó, se corroboró la necesidad de implementar la formación de posgrado extensionista. Con la entrevista se obtuvo un listado de conocimientos específicos necesarios y se constataron los intereses de los futuros cursistas. Los cursos demandados son mostrados en la figura 1.

\section{Figura 1. Temas demandados por los entrevistados}

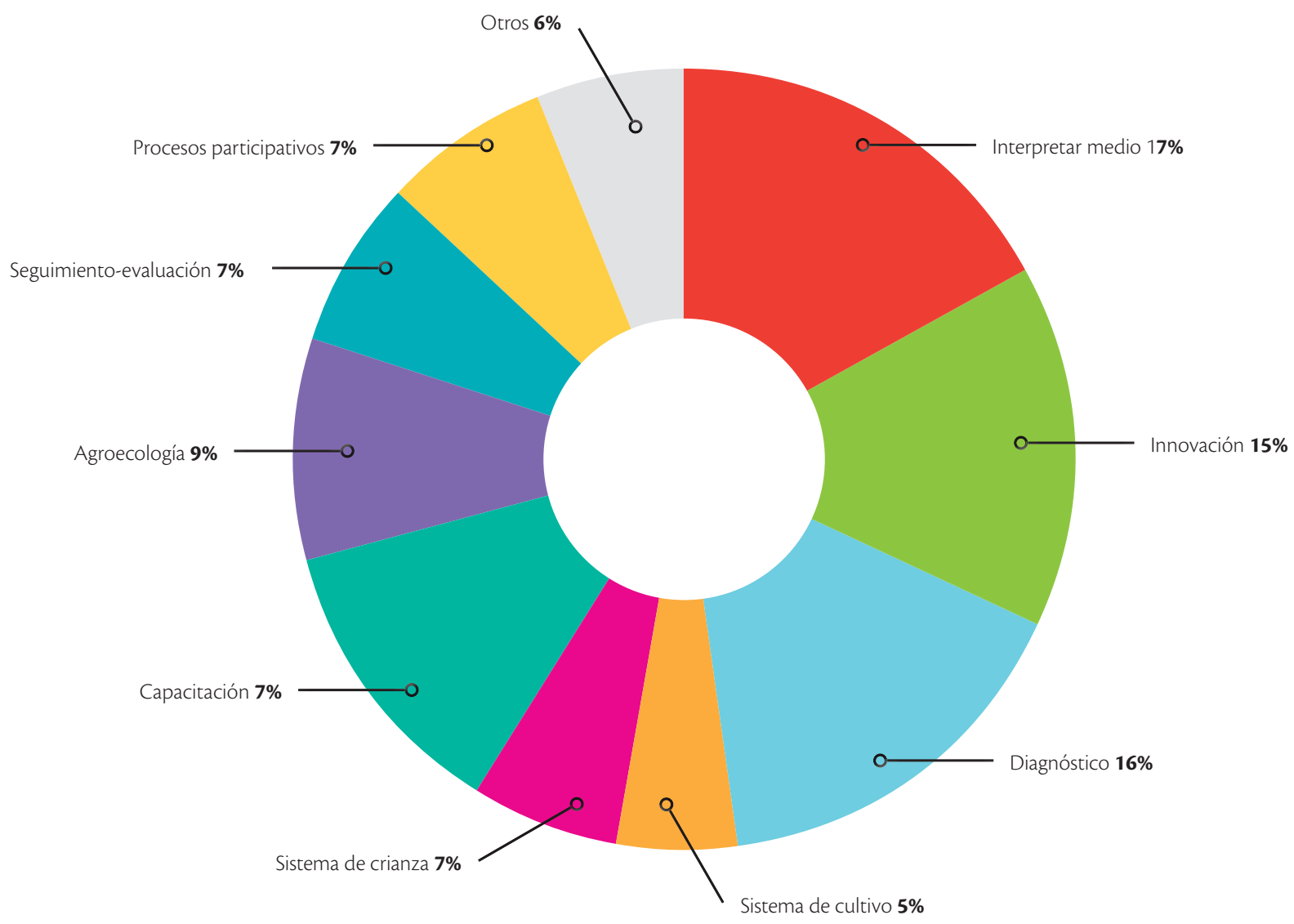


Los temas más demandados fueron: interpretación del medio agrícola y rural, diagnóstico, innovación rural y capacitación. Hubo temáticas menos solicitadas que guardan relación estrecha con la extensión agraria, excepto el 6\% que solicitó otros relacionadas con sistemas de crianza animal.

Como en la gestión del capital humano un área de resultados clave es la formación (Mir-Fruto, 2015), hubo 43 profesionales de la provincia Holguín que demandaron la matrícula en la Especialidad de Posgrado en Extensión Agraria. El mayor número de solicitantes fueron de la Unidad de Extensión, Investigación (UEICAH), a ello contribuyó la existencia de proyectos de investigación-desarrollo con asesoría francesa y la experiencia en el desempeño de la actividad de extensión agraria.

Como colofón para la primera edición de la Especialidad de Posgrado en Extensión se tuvieron en cuenta algunos elementos claves, los que se comentan a continuación: a) para responder a la problemática formativa, no basta con acciones, es necesario conocer la satisfacción de la sociedad (Álvarez-Herrera et al., 2013), sobre esta base fue sistematizado el proceso; b) las necesidades formativas de los extensionistas exceden los contenidos técnicos, incluyendo conocimientos referidos a comercialización, metodología de extensión rural, manejo de grupos y técnicas de trabajo participativas (Landini 2013), por lo que el programa contó con diversos cursos básicos y optativos.

Fase 3: identificación e importación a Holguín de la Especialidad de posgrado en Extensión Agraria (2014). Este posgrado fue iniciado como diplomado con los contenidos del programa idéntico al oficial para contribuir a las convalidaciones, con los 108 créditos correspondientes a la especialidad demandada. Para la adecuada publicidad de la convocatoria se divulgó por la radio provincial, también con un plegable y a través de instituciones involucradas en los encuentros.

\section{Desarrollo de los diplomados que conformaron la especialidad de posgrado (2014-2015)}

En este periodo se impartieron dos diplomados con 14 cursos y con un total de 108 créditos, desarrollados en dos años. El primer diplomado se nombró "Fundamentos, prácticas y metodología de la extensión para el desarrollo sostenible" y el segundo "Herramientas de la extensión agraria". En la tabla 1 se listan los nombres de los cursos.

El programa original de la UNAH contenía la propuesta de siete cursos optativos; Agroecología, Sistemas Agrícolas, Sistemas Pecuarios, Sistemas Forestales, Gestión de la Unidad de Producción, Sociología Agraria y Economía Agraria.

Tabla 1. Cursos de la Especialidad de Posgrado en Extensión Agraria

\begin{tabular}{|l|l|}
\hline \multicolumn{1}{|c|}{ Diplomado 1 } & Diplomado 2 \\
\hline 1. Marco nacional e internacional del agro cubano & 1. El diagnóstico \\
\hline 2. Enfoque y prácticas del desarrollo sostenible & 2. La adopción de innovaciones \\
\hline 3. Visión sistémica de la realidad agraria & 3. La gestión participativa de proyectos \\
\hline 4. Formas y roles de la extensión & 4. Negociación \\
\hline 5. Proceso general de la extensión para el desarrollo sostenible & 5. Sociología Agraria \\
\hline 6. Comunicación y educación popular & 6. Economía Agraria \\
\hline 7. Trabajo grupal y coordinación & \\
\hline 8. Capacitación rural & \\
\hline
\end{tabular}

Fuente: Programa de la Especialidad, Edición 1 en Holguín. 
En Holguín se mantuvo la propuesta de los cursos optativos concebidos en el programa original, y al iniciar el segundo diplomado se ofertaron a los participantes los cursos que por consenso los cursistas seleccionaron de los dos últimos diplomados. El argumento dado fue que en su mayoría trabajaban en centros agropecuarios donde escasamente se ofertan materias de otras disciplinas.

La matrícula fue de 31 cursistas matriculados con multiperfiles, egresados de carreras técnicas como las Ingenierías Agronómica, Pecuaria, de Riego y drenaje, y carreras de licenciaturas como Economía, Contabilidad, Pedagogía y Psicología.

La especialidad se organizó en 18 cursos y los profesores de cada uno lo diseñaron, compartieron y validaron en el marco de un taller con otros profesores, lo que permitió compartir criterios que favorecieron la impartición de contenidos.

Fase 4: análisis de los dos diplomados desde la sistematización de la experiencia. En cuanto a los cursos del Diplomado I: el 23\% considera que se lograron los objetivos en todos los cursos, el 70\% en algunos cursos y el $7 \%$ en pocos cursos; en cuanto a la relación entre los cursos, el 38\% la considera muy buena y el 62\% buena. Respecto a los cursos del Diplomado II "Las Herramientas de la Extensión Agraria", consideraron que contribuyen a su formación como extensionista en los siguientes aspectos: la adopción de herramientas, la adquisición de conocimientos, el desarrollo de habilidades para identificación de problemas y demandas, para impulsar el desarrollo agroecológico, apropiación de métodos y formas para educar y aprender, integralidad, apropiación de la sociología, profundización en temas de innovación y negociación, y para conocer sobre el trabajo en equipos y el intercambio de experiencias.

Se realizaron intercambios con los profesores principales de cada curso, de conjunto con la coordinadora general, a fin de compatibilizar y prever el solapamiento de contenidos. También para fortalecer el principio de preparar al pedagogo para un proceso participativo basado en la educación popular promulgado por Freire (1973) y por Ortiz (1998), cuando hacían alusión a la importancia de la interacción constante educadores-educandos.

En cada diplomado se desarrolló un taller intermedio y uno final, los que contribuyeron a la definición y perfeccionamiento de los temas de trabajo para el informe final. La evaluación general se hizo de forma cuantitativa con una norma de calificación de 5 puntos como máximo y 3 puntos como mínimo. Con la elaboración de una tesina individual se cerró el proceso de posgrado, en ella se mostraron elementos teóricos y prácticos desarrollados en la investigación. En la figura 2 se muestran las temáticas trabajadas en los informes finales. Se debe identificar bien lo que corresponde a metodología para incluirlo en ella.

Se desarrollaron temas en respuesta al interés de los estudiantes y de alta pertinencia para los centros de trabajos de procedencia de los diplomantes. El diagnóstico a escala municipal y de unidad de producción fue desarrollado por diez cursistas. La innovación participativa por nueve, en la que prevaleció la selección de variedades y de tecnologías.

Los planes de acción se definieron participativamente, dando un enfoque democrático a la difusión de la agroecología. Para las cooperativas agropecuarias se mostraron cinco informes finales.

La sistematización se hizo en tres tesinas para recoger la historia extensionista de un municipio que en este caso correspondió a Banes, de donde se recogieron las experiencias de la edición de la Especialidad para el proceso integral en una cooperativa de producción agropecuaria. El seguimiento, la evaluación y la proyección fueron temas de dos reportes sobre tecnología en fincas, principalmente en adopción de bionutrientes y la planeación estratégica de la extensión agraria provincial para el quinquenio 2015-2020 que quedó plasmada en un informe. 


\section{Figura 2. Temas de trabajo defendidos en la Especialidad}

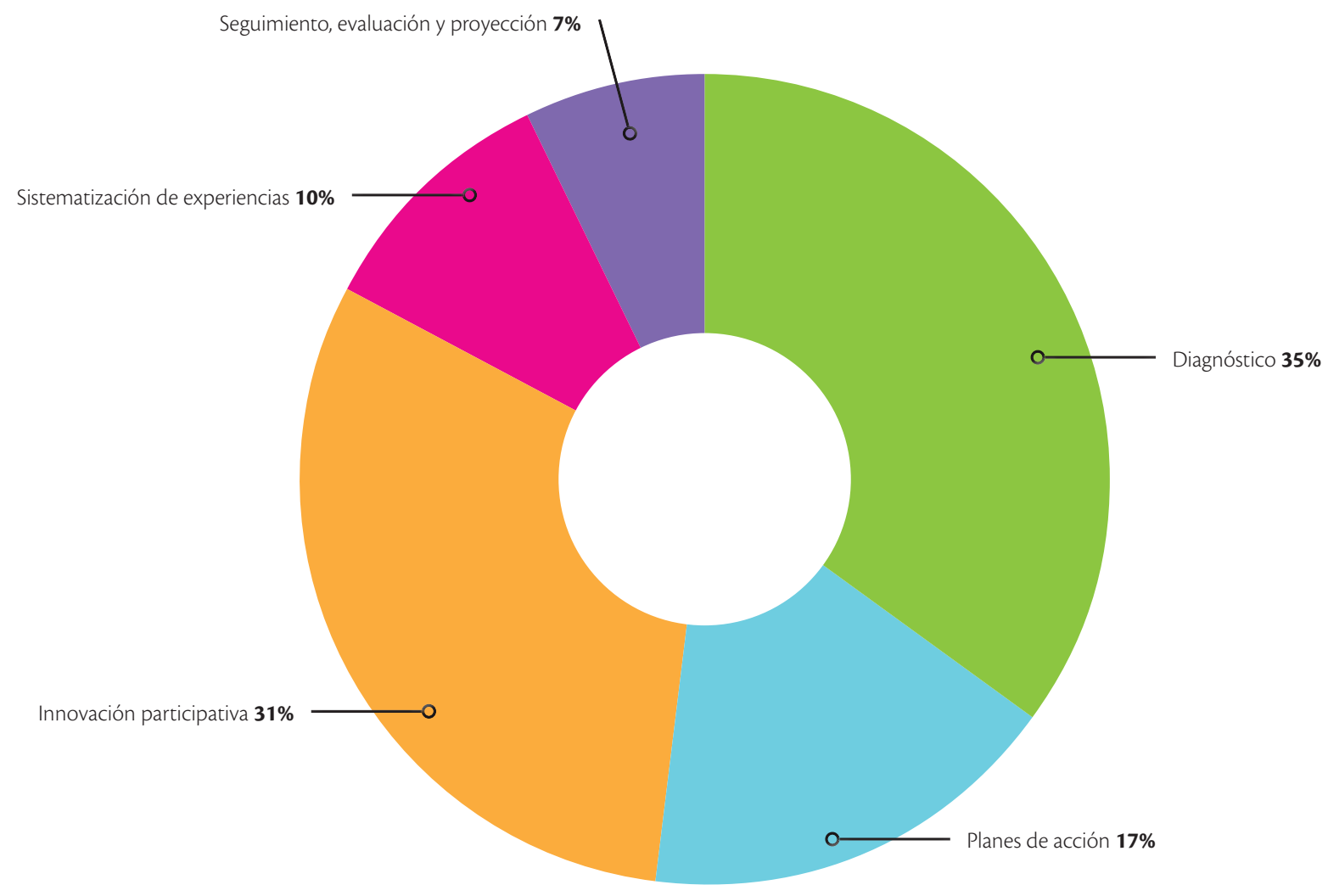

Fuente: de los autores, a partir de temas desarrollados, 2018

Fase 5: sistematización de experiencias con egresados (2016-2018). Para la sistematización del proceso de la edición I de la Especialidad, que representa el $94 \%$ del total matriculado, los mismos laboran en diferentes cargos en su desempeño profesional, como puede apreciarse en la figura 3.

El mayor número de egresados son especialistas de centros de investigación, fundamentalmente de la UEICAH en Holguín. Son personas vinculadas desde hace más de 10 años con la investigación sobre el cultivo de las viandas tropicales, las hortalizas y los pastos y forrajes. Los especialistas de las empresas pertenecen a: Granos Gibara, comercializadora de acopio provincial y delegaciones de la agricultura en Báguanos y en la granja urbana provincial. En el caso de los profesores, pertenecen a las carreras de Ingeniería en Agronomía y licenciatura en Economía, vinculados en todos los casos por más de cinco años a proyectos de investigación-desarrollo. Los extensionistas y los capacitadores trabajan en las direcciones municipales del Ministerio de Agricultura de Báguanos, Banes, Gueto, Urbano Noris y Gibara. Los cooperativistas son un agricultor y una presidente de unidad productiva. La categoría otros en el gráfico pertenece a una especialista de la Asociación Cubana de Producción Animal (ACPA) provincial y a un funcionario de la esfera agropecuaria en el municipio de Gibara. 


\section{Figura 3. Desempeño laboral de los egresados}

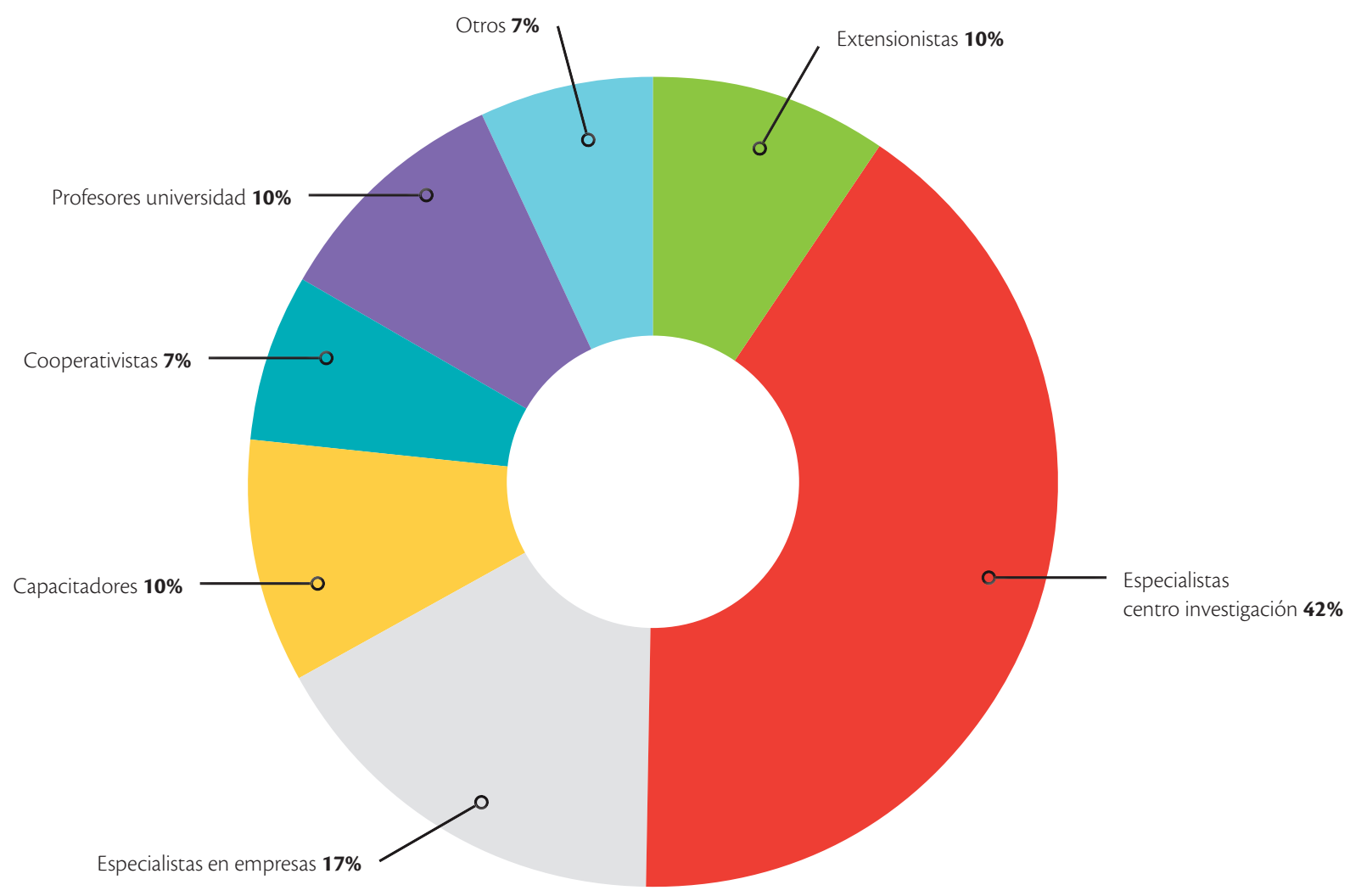

Fuente: elaboración de autores a partir de entrevistas, 2018.

Todos los graduados manifiestaron aplicabilidad de los conocimientos adquiridos en la práctica de su labor como profesionales. La figura 4 muestra dos áreas de desempeño y uso de una herramienta analítica con aplicabilidad de los conocimientos adquiridos por los egresados durante su formación extensionista.

En la figura 4 se constata que en el $43 \%$ hay aplicabilidad en procesos participativos y de capacitación. Se ha llevado a cabo la identificación de demandas de capacitación en contacto directo con agricultores en el marco de reuniones de cooperativas agropecuarias y mediante visitas a fincas. Los temas a capacitar han sido diversos, tanto de la rama agropecuaria como de otras materias, destacándose materias como el crédito, los seguros y los contratos de comercialización, extendiéndose por todos los municipios de procedencia de los graduados. Promover la participación ha sido un útil aprendizaje que ha facilitado procesos en el propio entorno laboral, según aseveran los egresados, así como en trabajos in situ en la agricultura. Es alentador este elemento si se considera que una de las dificultades de los extensionistas es trabajar en grupo, por insuficientes habilidades y tradición. Además, aún es bajo el acceso a asistencia técnica y capacitación de los productores agrícolas en el contexto holguinero, lo cual también se pone de manifiesto en otras regiones del mundo, según reportan autores como la FAO (2014), Landini (2015) y Š̄mane et al. (2017). 


\section{Figura 4. Aplicabilidad de conocimientos adquiridos}

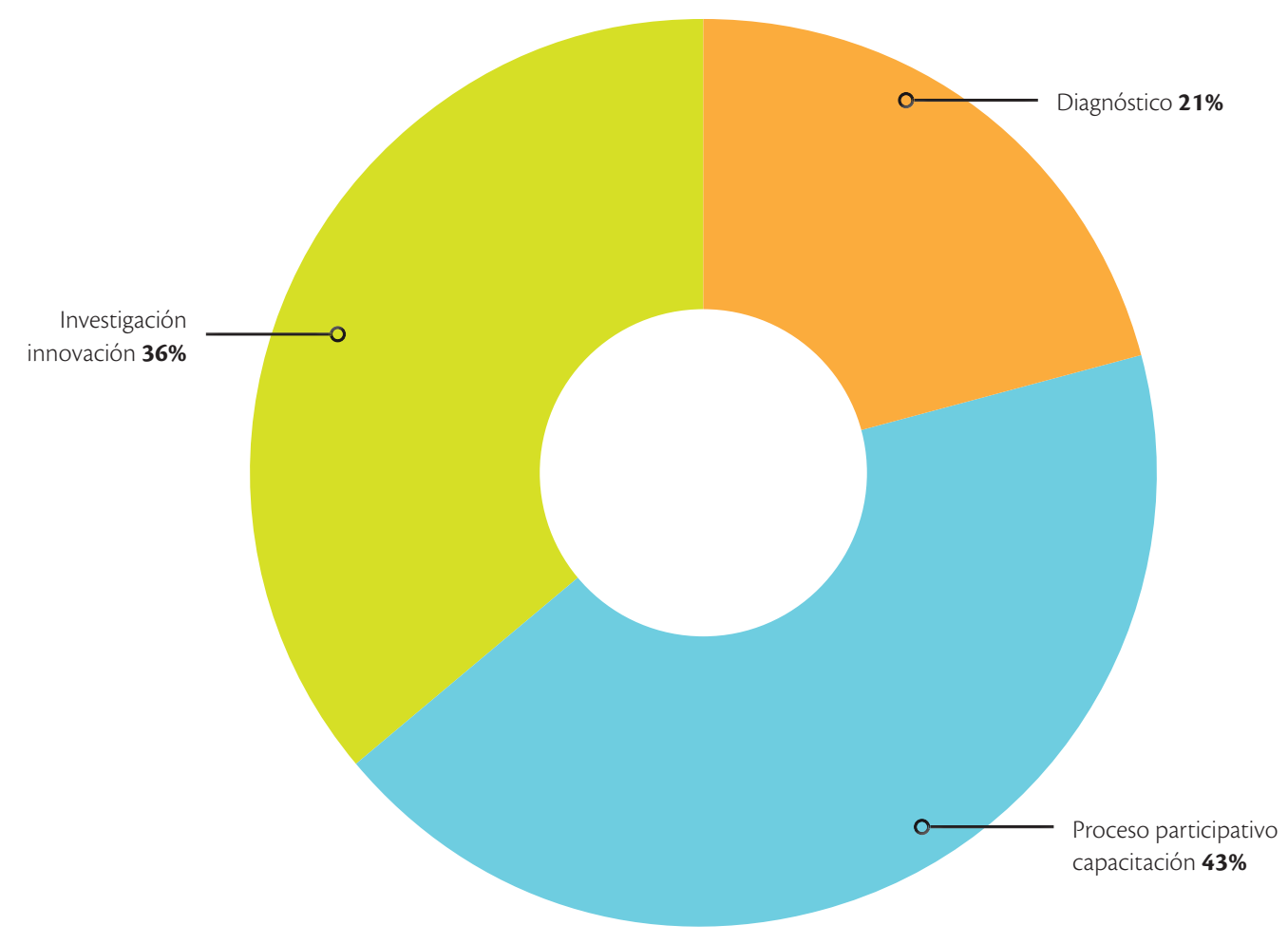

Fuente: elaboración de autores a partir de entrevistas, 2018.

El 36\% de los cursistas ha aplicado conocimientos en la investigación y la innovación, en correspondencia con el perfil de desempeño de egresados que están presentes en transferencia de variedades y de tecnologías con agricultores. Existen especialistas de empresas, extensionistas y capacitadores que se han involucrado en el proyecto nacional de fitomejoramiento participativo en comunidades agrarias. Es significativa la cadena de valor del cultivo del frijol en los municipios de Gibara, Báguanos, Cueto; las prácticas agroecológicas en Banes, Báguanos y las variedades de viandas tropicales diseminadas por toda la provincia.

También directivos de los organismos empleadores de los graduados manifestaron alta satisfacción con la contribución a la innovación participativa y el enfoque sistémico. Sin embargo, no se debe perder de vista que es una tarea compleja transformar las prácticas extensionistas, según Landini (2014). Asimismo, el papel de los sistemas de extensión agraria concebidos para comunicar y diseminar nuevas prácticas agrícolas, no son suficientes para hacer frente a muchos de los nuevos desafíos (Terblanche, 2017). La herramienta de análisis más utilizada es el diagnóstico, seguido de la definición de planes de acción aplicado por el 21\% de los cursistas, lo cual ha sido utilizado en todos los municipios para sistemas de cultivo y de crianza, y de unidades de producción. También los planes de acción han sido derivados de los diagnósticos a diferentes niveles, creándose una 
lógica de identificación de problemas y propuestas de solución. El desafío es que se consideran esenciales dos premisas, que sea un proceso participativo y con un pensamiento sistémico. Así, debe abordar aspectos sociales, técnicos, medioambientales, históricos, organizativos y económicos relacionados con la producción agropecuaria. Esta visión facilita enfocar acciones de desarrollo con mayor objetividad.

También los profesionales con cargo de extensionista valoran la incorporación de análisis diagnósticos a más profundidad, como es el caso de la construcción de tipologías. La continuidad de aplicación de esta herramienta deberá tener en cuenta la situación que se trate, abundancia o escasez de datos disponibles para clasificar fincas y la factibilidad de realizarla (Paas y Groot, 2017). Se considera que los resultados de la aplicabilidad de conocimientos adquiridos por parte de los titulados se han llevado a la práctica tanto por profesionales de ocupación laboral extensionista como por otros que trabajan en otras ocupaciones en el entorno agrario.

\section{Algunas reflexionesde los autores \\ A partir del programa de la especialidad de posgrado en Holguín (edición 1)}

La formación posgraduada extensionista presenta limitantes que ameritan ser resueltas, por lo que se ha considerado el proceso de la edición I de la especialidad en Holguín para hacer propuestas. El orden y contenido de los cursos se ha mantenido idéntico al programa original por lo que se imponen versiones adaptadas al contexto donde se impartirán. Landini (2014) ha profundizado en la necesidad del enfoque territorial y orientado a la articulación interinstitucional.

- Se debe invertir la forma de impartición otorgando un $80 \%$ a la práctica y $20 \%$ a la teoría, saliendo del escenario del aula hacia el campo y vinculando los temas de informe a las clases prácticas que se realicen.
- Hasta el momento dentro del programa algunos temas no han sido tratados: el uso de las tecnologías de información y comunicación; el trabajo en redes o colaboración multiactores, existiendo potencialidades por cursos impartidos por investigadores belgas (Craps y Sips, 2014); metodologías de investigación acción; profundización en técnicas de participación con agricultores; nuevos métodos estadísticos, porque se incursiona en investigaciones con un cambio de paradigma de la experimentación en condiciones controladas a la observación multivariada.

- El tema de informe para la culminación de estudios debe definirse desde los primeros encuentros, estar vinculado a las clases prácticas y planificarlo por etapas hasta llegar al informe final. En la actualidad no se ha tomado en cuenta una planificación con tareas distribuidas durante todo el periodo de la especialidad, lo cual ha traído como consecuencia que en algunos casos no se cuente con suficiente tiempo para implementar un proceso de extensión agraria, en caso de que corresponda de acuerdo con el tema seleccionado.

- Ha sido limitada la incorporación de investigaciones en trabajos finales que relacionen el apoyo de la psicología con el ámbito pedagógico o comunicacional para contribuir a cambiar la mentalidad de los productores. En este sentido, Landini (2016a) corrobora estas necesidades en investigación realizada con extensionistas cubanos.

\section{Reflexiones generales en torno a la actividad de extensión agraria}

El nuevo modelo de la economía cubana, plasmado en los Lineamientos de la Política Económica y Social del Partido y la Revolución Cubana de 2016, impone cambios sustanciales en todos los sectores. Por lo que en el análisis de la actividad extensionista surgen nuevos retos: 
- Adaptación a la diversificación de las demandas.

- La adaptación a nuevas demandas dependerá de los modelos agrícolas, de las características de los agroecosistemas, las expectativas del consumidor y la organización de las cadenas productivas. Como planteara Marzin (2017), hay que pasar de la recomendación genérica a la identificación de soluciones específicas para numerosos tipos de entornos agroecológicos y socioeconómicos, así como de nuevas articulaciones entre actores de la innovación.

- Generación, adaptación y utilización de nuevos conocimientos. Esta cuestión fue tratada por Terblanche (2017) para el abordaje de retos globales, el autor coincide que esto implica la interacción y apoyo de una amplia gama de organizaciones en el sistema de innovación agrícola y el acuerdo entre múltiples actores.

- La multifuncionalidad de la agricultura. En el contexto holguinero, con el crecimiento del turismo, se impone dar valor a las potencialidades que poseen los agricultores y abrir nuevos campos en este sentido implica ampliar las competencias de los extensionistas e incluir nuevas funciones. Wang et al. (2016) han abordado estas cuestiones, al resaltar el papel de espacios rurales en el paisaje y la valoración de éstos por el Estado. También Royo-Márquez et al. (2018) valoran que la extensión agraria es también vínculo con los productores y conexión interdisciplinaria para la acción, pero necesita impulsar el desarrollo local, dado que la producción agropecuaria se concibe desde un punto de vista multifuncional; Brinkley (2017) plantea la fortaleza de la inclusión de las actividades agrícolas dentro del paisaje suburbano.

- Cambio de enfoques y paradigmas. Esto es un reto grande, porque la extensión es una disciplina científica que nació prioritariamente como un instrumento conceptual y metodológico para sustentar el proyecto de modernización e industrialización de la agricultura, según confirmaba Clavijo (2015), pero han aparecido nuevos roles en lo nacional e internacional.

- Necesidad de completar el ciclo de formación posgraduada en Extensión Agraria con la incorporación de programas nacionales de doctorado y posdoctorales.

- Activar el Sistema Nacional de Extensión Agraria (SEA) en el país y los territorios de la geografía nacional, puesto que en las condiciones actuales es limitada la forma de extensión integral.

- Activación de los grupos municipales de extensión agraria y la creación de grupos focales de discusión monitoreados por éstos. Se podrían aprovechar espacios para compartir las experiencias sobre alternativas de producción agrícola, como las planteadas por Peña-Borrego et al. (2018). Esos espacios son: las asambleas de asociados, conferencias en la base de cooperativas, talleres, conversación con campesinos, en el campo, ferias, cursos de la universidad y en visitas a productores.

- Necesidad de publicar experiencias de sistematización de procesos formativos (ediciones de posgrados, especialidades y maestrías) y de investigaciones puras sobre extensión agraria e investigaciones de diferentes ámbitos, llevadas a cabo en agroecosistemas desde diferentes metodologías.

- Construcción de propuestas metodológicas extensionistas, contextualizadas en los territorios.

- Repensar las competencias laborales del cargo de extensión agraria y reactivarlo en el profesiograma nacional. Sobre las competencias del agente de extensión agraria, Rodríguez-Armán et al. (2017) afirman que son vitales la comunicación, orientación al servicio y trabajo en equipos y la gestión de recursos humanos. 


\section{Conclusiones}

Las demandas formativas para el posgrado en Extensión Agraria en el territorio holguinero han recibido una respuesta positiva, lo cual fue constatado en esta investigación con profesionales egresados de los cursos desarrollados. La sistematización del proceso de posgrado extensionista permitió identificar limitantes y perspectivas tanto en la formación como en la actividad de extensión agraria.

\section{Referencias}

Aguirre-Vélez, J., F. Castrillón-Hernández y B. ArangoAlzate (2019), "Tendencias emergentes de los posgrados en el Mundo", Revista Espacios, vol. 40, núm. 31, pp. 9-23.

Almaguer-Pérez, N. (2015), "Diagnóstico Agrario del municipio Calixto García, actualización año 2014”, Tesis presentada en opción al Especialista, Holguín, Universidad de Holguín.

Almaguer-Pérez, N. (1996), "Análisis del VII Curso Internacional de Extensión y Desarrollo Rural impartido en la Escuela Central de Capacitación Agraria y su aplicación a las condiciones de Cuba", en Memorias VII Curso Internacional de Extensión y Desarrollo Rural, Madrid.

Álvarez-Herrera, D., J. Cejas-Montero, D. AlfonsoRobaina y A. Alonso-Becerra (2013), "Modelo de gestión universitaria para las áreas de resultados clave del Instituto Superior Politécnico José Antonio Echeverría”, Gestión Universitaria, vol. 5, núm. 3, pp. 14-22.

Brinkley, G. (2017), "Visualizing the social and geographical embeddedness of local food systems", Fournal of Rural Studies, vol. 54, núm. 3, pp. 314-325.

Caballero, R.-A., A. Casanova, A. Hernández y J. F. Capote (2000), "La asistencia técnica a los productores
Se revelaron deficiencias en las políticas relacionadas con la implementación del Sistema de Extensión Agraria nacional, lo que repercute en los territorios tanto en la formación como en formas integrales de trabajo.

Los egresados de la primera edición de la Especialidad de posgrado en Extensión Agraria han aplicado los conocimientos adquiridos, lo que se constata en la práctica en su entorno laboral.

en Cuba. Conceptos y evolución", Cuadernos de Desarrollo Rural, núm. 45, pp. 91-104.

Castro-Rubilar, F., H. Lira-Ramos y M. T. Castañeda (2017), "Estudio evaluativo del diseño e implementación curricular de la formación pedagógica en carreras de educación”, Revista Actualidades Investigativas en Educación, vol. 17, núm. 2, mayo-agosto, pp. 1-23.

Cid-Lazo, G. (2017). "Investigación agronómica y Extensión Agraria en Cuba: unidad dialéctica imprescindible para lograr seguridad alimentaria", Revista Ingeniería Agrícola, vol. 3, núm. 3, pp. 35-38, <https://revistas.unah.edu.cu/index.php/IAgric/ article/view/620>.

Clavijo, N. (2015), "Antecedentes y nuevas perspectivas de la extension rural en Colombia", en I Congreso Latinoamericano de Teoría Social, Buenos Aires, Instituto de Investigaciones Gino Germani, Facultad de Ciencias Sociales, Universidad de Buenos Aires.

Craps, M. y Sips, K. (2014), "Facilitando la colaboración entre múltiples actores para la sustentabilidad rural", ponencia presentada en el Taller de inicio de proyecto Holguín, Holguín.

Cruz,V.(2014), "TendenciasdelposgradoenIberoamérica”, Ciencia y Sociedad, vol. 39, núm. 4, pp. 641-663. 
Culebro-Trujillo, R., A. García-Villaseñor, R. BarragánHervella, A. Montiel-Jarquín, G. López-Cázares, S. Romero-Figueroa y C. Medina-Escobedo (2017), "Evaluación del plan de estudios para la formación de bibliotecarios en Ciencias de la Salud, una nueva propuesta", Investigación Bibliotecológica, vol. 31, núm. 71, enero/abril, pp. 219-234.

Engel, P. (2001), Facilitando el desarrollo sostenible: ¿hacia una extensión moderna?, Santiago de Chile, CEDRO.

Enrique-Alemany, C. (2012), "Elementos para el estudio de la dinámica y evolución histórica de la extensión rural en Argentina", tesis, Doctor en Ciencias, Córdoba, Universidad de Córdoba.

FAO (2014), Agricultura familiar en América Latina y el Caribe. Recomendaciones de política, Latinoamerica y Caribe, FAO <http://www.fao.org/fileadmin/user_upload/AGRO_ Noticias/docs/RecomendacionesPolAgriFAMLAC.pdf>.

Faure, G., K. Huamanyauri-Méndez, I. Salazar, C. Gómez, E. de-Nys y M. Dulcire (2015), "La privatización del asesoramiento agrícola: consecuencias para los productores lecheros del valle del Mantaro, Perú", Cuadernos de Desarrollo Rural, vol. 12, núm. 76, pp. 12-22, DOI: http://dx.doi.org/10.11144/Javeriana. cdr12-76.paac.

Freire, P. (1973), ¿Extensión o comunicación?, México, Siglo XXI.

Gaceta Oficial de Cuba (2019), "Resolución 140/19", Reglamento de la educación de posgrado de la República de Cuba, La Habana, GOC (2019-776-O65).

Henríquez, P. (2018), "Sondeo de prioridades de los institutos nacionales de investigación agrícola en las Américas", Informe al Foro de las Américas para la Investigación y Desarrollo Tecnológico Agropecuario, EUA, Instituto Interamericano de Cooperación para la Agricultura.

Hernández-Gil, J., G. Figueroa, J. Núñez-Jover, I. Armas y A. Alcázar (2014), “Obstáculos al desarrollo local en Cuba. Análisis y propuestas desde la gestión universitaria del conocimiento y la innovación", en Jorge Nuñez-Jover, Universidad, conocimiento e innovación, La Habana, Ed. Félix Varela, pp. 55-60.
Horrutinier P. (2007), "El proceso de formación. Sus características", Pedagogía Universitaria, vol. 12, núm. 4.

Klerkx, L., F. Landini y H. Santoyo-Cortés (2016), "Agricultural extension in Latin America: current dynamics of pluralistic advisory systems in heterogeneous contexts", The fournal of Agricultural Education and Extension, vol. 22, núm. 5, pp. 389397, DOI: http://dx.doi.org/10.1080/138922 4X.2016.1227044 [Consulta: octubre de 2019].

Landini, F. (2016a), "La concepción de extensión rural en 10 países latinoamericanos", Revista de Investigación Social, <https://www.redalyc.org/service/redalyc/ downloadPdf/628/62845862011/6> [Consulta: octubre de 2019].

Landini, F. (2016b), "Diversidad de expectativas de los extensionistas rurales latinoamericanos sobre la psicología”, Estudios de Psicología, 21(4), pp. 8-18.

Landini, F. (2016c), “Concepción de extensión rural en 10 países latinoamericanos", Revista de Investigación Social, DOI: 10.29092/uacm.v13i30.9.

Landini, F. (2015), "Problemas enfrentados por extensionistas rurais brasileiros e sua relação com suas concepções de extensão rural", Ciência Rural, vol. 4, núm. 2, pp. 371-377, DOI: 10.1590/01038478cr20140598.

Landini, F. (2014), Desafios para la transformación de los enfoques y prácticas de los extensionistas rurales latinoamericanos desde una perspectiva psicosocial, Buenos Aires, 5th GFRAS Annual Meeting.

Landini, F. (2013), "Necesidades formativas de los extensionistas rurales paraguayos desde la perspectiva de su función, sus problemas y sus intereses", Mundo Agrario, núm. 20, pp. 149-160.

Landini, F. y L. Riet (2015), "Extensión rural en Uruguay: problemas y enfoques vistos por sus extensionistas", Mundo Agrario, vol. 16, núm. 32.

Marzin, J, A. Solene-Benoit, V. López, G. Cid, V. Peláez, N. Almaguer, A. Herrera y M. Mercoire (2014), Herramientas metodológicas para una extensión agraria generalista, sistémica y participativa. Proyecto de apoyo al Sistema de Extensión Agraria en Cuba (ISBN: 978-959-7210-70-2.2014), La 
Habana, Editora Agroecológica, <http://agris.fao.org/ agris-search/search.do?recordID=FR2017103635> [Consulta: septiembre de 2019].

Marzin,J. (2017), "Desafios y perspectivas de la Extensión Agraria en un mundo más complejo", Congreso Internacional de las Ciencias Agropecuarias, Agrociencias, noviembre, La Habana.

Marzin, J., T. López y G. Cid (2003), “Tendencias actuales en transferencia de tecnologías y extensionismo. Lecciones para la situación en Cuba”, ponencia en el Fórum Especial Nacional de Extensión Agraria, La Habana, MINAG.

Matías-González, A. (2012), "Ensayo crítico sobre el cooperativismo en Cuba", Revista Acdémica de Economía, Observatorio de la Economía Latinoamericana, núm. 135.

McMahon, M. A., Valdés, A., Cahill, C. y Jankowska. A. (2011), Análisis del extensionismo agrícola en México, París, Organización para la Cooperación y el Desarrollo Económico (OECD).

Ministerio de la Agricultura (MINAG) (2011), Estadísticas Oficina Control Tierra y Tractores, Holguín.

Mir-Frutos, Z. (2015), "Planeación estratégica del Sistema de Extensión Agraria 2016-2018”, Trabajo final presentado en opción al Título Académico de Especialista de posgrado en Extensión Agraria, Holguín, Universidad de Holguín.

Miranda-Agüero, Y., N. Arozarena y T. López (2016), "Extensión Agraria en la percepción de profesores de la Universidad Agraria de La Habana", Revista de Gestión del Conocimiento y el Desarrollo Local, vol. 3, núm. 1, pp. 10-18.

Novo, R. (2011), Orígenes y desarrollo de la agricultura y de los estudios agrícolas en Cuba: apuntes para una historia, La Habana, Félix Varela.

Ortiz, E. (1998), "Comunicación pedagógica y aprendizaje escolar", Revista Cubana de Psicología, vol. 18, núm. 2, pp. 141-146.

Paas, W. y J. Groot (2017), "Creating adaptive farm typologies using Naive Bayesian classification", <www. elsevier.com/locate/inpa, from www.sciencedirect. com> [Consulta: septiembre de 2019].
Pavón, J. (2015), "Principales resultados para la implementación del sistema de gestión de Extensión Agraria en el municipio Güines, provincia Mayabeque", Cultivos Tropicales, núm. 36, pp. 17-22, $<$ http://scielo.sld.cu/scielo.php? script=sci_arttext\&p id=S0258-59362015000100002 $>$ [Consulta: octubre de 2019].

Peña-Borrego, M.-D., Y. Peña-Rueda y N. Almaguer-Pérez (2018), "Necesidades de información y comportamiento informativo de agricultores y ganaderos en una CCS del municipio Calixto García", Maestro y Sociedad, núm. Especial III Taller Científico Metodológico de Información Científica 2018, pp. 158-164.

Pontes-Furtado, E. y J. Ribamar-Furtado De Souza (1998), "A intervenção participativa dos atores: Uma metodología construída no contexto dos assentamentos rurais do ceará (INPA)", Educação em Debate, vol. 20, núm. 36, pp. 154-162.

Riera, O. y J. Swinnen (2016), "Cuba's agricultural transition and food security in a global perspective", Applied Economic Perspectives and Policy, vol. 38, núm. 3, pp. 413-448.

Rodríguez-Armán, M., Z. Salguero-Rubio y A. GinebraAguilar (2017), "Las competencias laborales del cargo del agente de Extensión Agraria", Revista de la Facultad de Agronomía, vol. 116, número especial, pp. 39-44.

Rodríguez-González, N., N. Almaguer-Pérez y A. SerranoSantiesteban (2014), "The post-graduated formation in Agrarian Extension in Holguín, ITS contribution to the sustainability of agricultural systems", ponencia en la VI Edición de la Conferencia Científica Internacional sobre Desarrollo Agropecuario y Sostenibilidad "AGROCENTRO 2014", Villa Clara, Cuba.

Rodríguez-Lugo, B., N. Nápoles-Hernández, T. PérezCastro y N. Ramírez-Gómez (2016), "Socialización del conocimiento sobre Extensión Agraria en las revistas agrícolas certificadas en Cuba (2004-2014)", Revista Publicando, vol. 3, núm. 6, pp. 48-60.

Royo-Márquez, M.-H., S. Sierra-Tristán, J. RonquilloAboite, J. Ochoa-Rivero y R. Vázquez-Gómez (2018), "Análisis del componente extensionista en el norte de 
México", Revista Mexicana de Ciencias Agrícolas, vol. 9, núm. 4, <https://cienciasagricolas.inifap.gob.mx/ editorial/index.php/agricolas/article/view/1398> [Consulta: diciembre de 2018].

Sablón-Pérez, A.-M., J. Marzin, R. Caballero, Z. Salguero-Rubio, T. López-Betancourt, Y. VallejoZamora, N. Ramírez-Palma, A. Marrero y G. CidLazo (2012), Memoria de los talleres nacionales de Extensión Agraria, La Habana, Agroecológica (ISBN: 978-9597210-62-7).

Salguero-Rubio,Z., A. Sablón-Pérezy T.López-Betancourt (2018), "La extensión agraria en la educación superior en Cuba", Pedagogía Universitaria, vol. XXIII, núm. 1, pp. 16-28.

Salguero-Rubio, Z., J. Marzin, A. Taboada-Zamora, A. Sablón-Pérez y R. Valdés-Carmenate (2016), "Las maestrías de la Facultad de Agronomía en la Universidad Agraria de La Habana desde la visión de sus profesores y estudiantes", Pedagogía Universitaria, vol. 21, núm. 3, pp. 1-22.

Sánchez-de-Puerta-Trujillo, F. (1996), Extensión Agraria y desarrollo rural, Madrid, Ministerio de la Agricultura Pesca y Alimentación.

Šūmane, S., I. Kunda, K. Knickel, A. Strauss, T. Tisenkopfs e I. Rios (2017), "Local and farmers' knowledge matters! How integrating informal and formal knowledge enhances sustainable and resilient agricultura", Journal of Rural Studies, vol. 59, pp. 232-241.

Terblanche, S. (2017), Advancing agricultural knowledge: improving the professionalism of rural advisory services, Lausanne, Switzerland, GFRAS.

Vargas-Leyva, M.-R. (2008), Diseño curricular por competencias, México, Asociación Nacional de Facultades de Ingeniería. Vázquez, L., N. González y J. Marzin (2017), "Políticas públicas y transición hacia la agricultura sostenible sobre bases agroecológicas en Cuba", en Red PPAL Políticas públicas a favor de la agroecología en América Latina y El Caribe, Brasilia, FAO, Red PP-AL, pp. 109132, <http://agritrop.cirad.fr/585704/> [Consulta: noviembre de 2019].

Vázquez-Moreno, L. (2008), "Desarrollo agroecológico adopción tecnologías y extensión; manejo agroecológico plagas de Cuba", Revista Brasileira de Agroecologia, vol. 3, núm. 1, <http://revistas.aba-agroecologia.org. br/index.php/rbagroecologia/article/view/6516> [Consulta: octubre de 2019].

Wang, C., B. Huang, C. Deng, Q. Wan, L. Zhang y Z. Fei (2016), "Rural settlement restructuring based on analysis of the peasant household symbiotic system at village level: a case study of Fengsi Village in Chongqing, China", fournal of Rural Studies, <https:// www.sciencedirect.com/science/article/pii/ S0743016716301231> [Consulta: junio de 2019].

\section{Cómo citar este artículo:}

Rodríguez-González, Niurlys, Nelvis-Alipio Almaguer-Pérez y José-Manuel García-Arias (2021), "Formación de posgrado en Extensión Agraria: experiencias en Holguín, Cuba", Revista Iberoamericana de Educación Superior (RIES), vol. XII, núm. 33, pp. 158-178, DOI: https://doi.org/10.22201/iisue.20072872e.2021.33.863 [Consulta: fecha de última consulta]. 